\title{
Frontal cutaneous meningioma - Case report*
}

\author{
Leonor Ramos ${ }^{1}$ \\ José Carlos Cardoso ${ }^{1}$ \\ Margarida Robalo Cordeiro ${ }^{1}$
}

\author{
Ines Coutinho ${ }^{1}$ \\ Helena Garcia ${ }^{1}$
}

DOI: http:/ / dx.doi.org/10.1590/abd1806-4841.20153798

\begin{abstract}
A bstract: Cutaneous meningiomas are rare tumors most commonly located on the scalp. We report the case of a 55-year-old male who presented with a $2 \times 3 \mathrm{~cm}$ tumoral lesion on the forehead. The lesion was hard, adherent and covered by normal skin. Incisional biopsy revelead a proliferation of monomorphic round cells, organized in nests and focally forming pseudovascular spaces. Immunohistochemical study revealed positivity for epithelial antigen membrane and vimentin. Vascular markers, cytokeratins and S100 protein were negative. A brain CT scan did not show any evidence of intracranial meningioma. The authors describe the case of a cutaneous frontal meningioma in probable relation with previous cranioencephalic trauma.
\end{abstract}

Keywords: Histology; Immunohistochemistry; Meningioma

\section{INIRODUCTION}

Meningioma is the most common tumor of the neuroaxis and is derived from meningothelial cells. Cutaneous meningiomas are collections of ectopic meningothelial cells in the dermis and subcutis, occuring most commonly on the scalp. ${ }^{1,2}$

The authors present a case of cutaneous meningioma of the frontal region with an unusual presentation and which could not be included into any of the classical types described. The objective of the report is to emphasize the rarity of this entity but also to increase awareness of its existence, so that clinicians can include it in the differential diagnosis of unusual scalp lesions.

\section{CASE REPORT}

A 55-year-old male presented with a slowly growing asymptomatic tumoral lesion in the frontal area, which had been evolving for 3 years.
Physical examination revealed a $2 \times 3 \mathrm{~cm}$, firm, ovoid subcutaneous mass, which was adherent to the deep tissues and covered by normal skin (Figure 1). There was no evidence of cervical lymphadenopathy on palpation.

The patient had suffered an occupational injury resulting in head trauma (24 years earlier) with fracture of the frontal bone and damage to the right brachial plexus. As a consequence, he developed encephalomalacia, right arm palsy and epilepsy (focal right frontal paroxysmal activity). In 2007 he suffered another episode of head trauma, which caused an acute fronto-parietal subdural hematoma. There was history of alcohol abuse. He was being treated with levetiracetam $1 \mathrm{~g} 2 \mathrm{id}$, oxazepam $15 \mathrm{mg}$ id and folic acid $5 \mathrm{mg}$ id.

A deep biopsy was performed and histological examination showed a proliferation of cells arranged in nests and lobules (Figure 2A). Some areas showed 

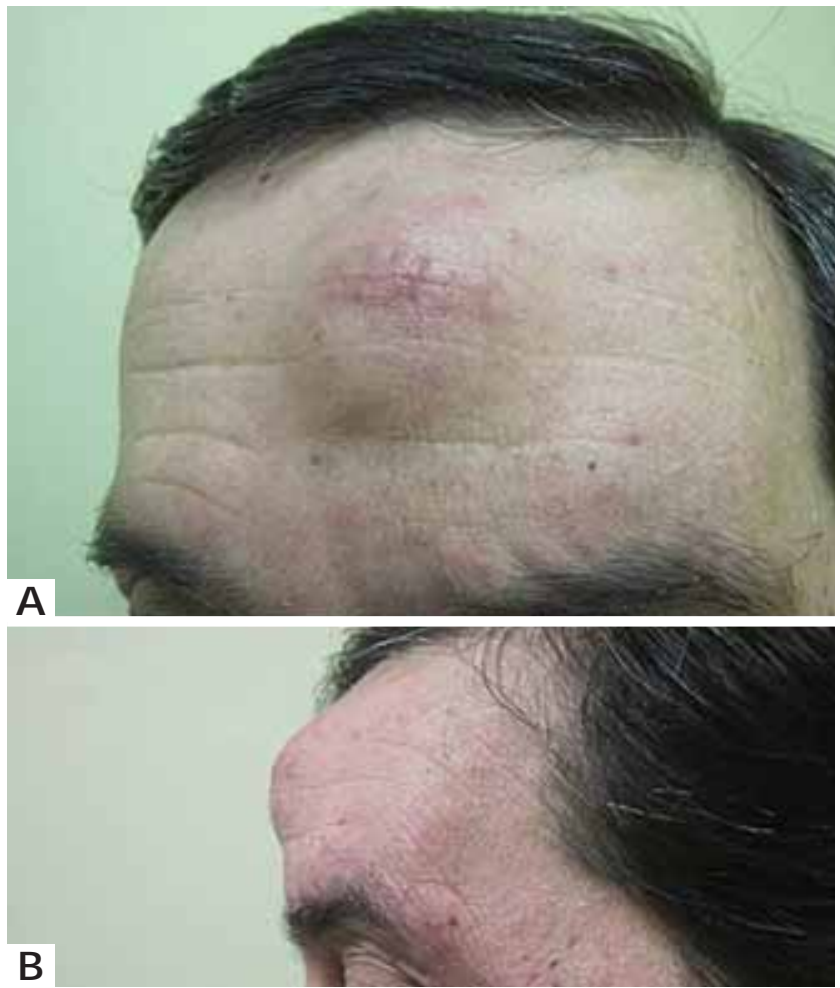

Figure 1: $2 \times 3 \mathrm{~cm}$, firm, ovoid subcutaneous mass, adherent to the deep tissues and covered by normal skin in the frontal region an interstitial growth pattern, with collagen dissection, evoking the formation of vascular spaces (Figure 2B). There was occasional entrapment of hyaline collagen, forming rounded collagen structures, but no calcification was seen (Figure 2C). The cells were round or ovoid, monomorphic, and devoid of mitotic activity or significant cytological atypia (Figure 2D). The nuclei were basophilic with finely scattered chromatin and the cytoplasm was moderately abundant, staining with a pale eosinophilic color.

Immunohistochemical study showed that the cells were positive for EMA and vimentin (Figure 3). Staining for epithelial (cytokeratins), melanocytic (S100 protein), vascular (CD34 and CD31), histiocytic (CD68) and muscular (desmin and smooth muscle actin) markers was negative. The combination of morphological and immunohistochemical features established the diagnosis of cutaneous meningioma.

Cranial computed tomography revealed a frontal mass and a bony defect in the frontal area (Figure 4 ). There were lesions of encephalomalacia in the frontal region and ventricular asymmetry (which had already been detected in previous exams) but no intracranial mass was detected.

The patient is now waiting for neurosurgical intervention.

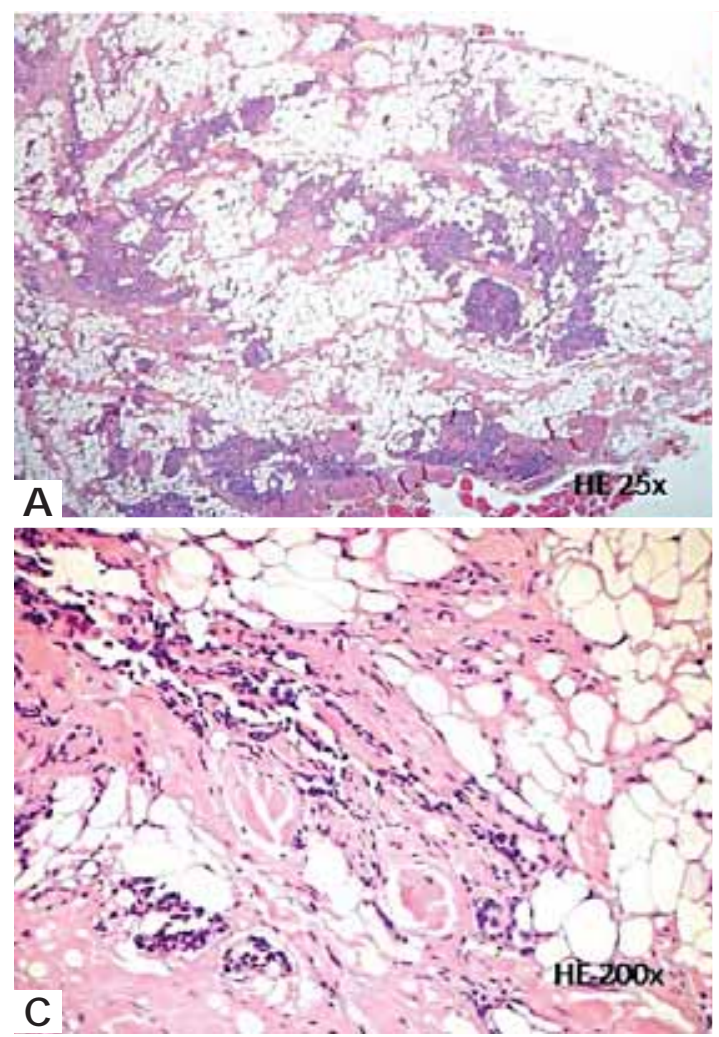

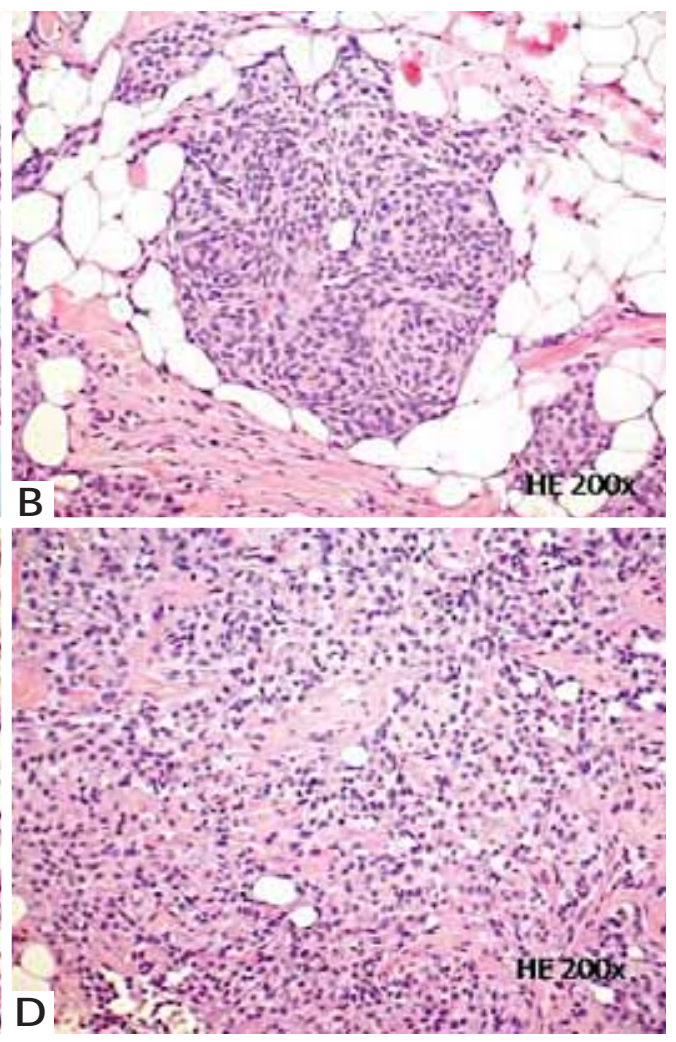

Figure 2: Histopathology of the lesion (HE stain) A. Proliferation of cells in lobular arrangement between adipose and muscular tissue. B. Proliferation of cells arranged in nests and lobules. C. Some areas presented intersticial growth, with collagen dissection, evoking the formation of vascular spaces. Areas of entrapment of hyaline collagen, forming rounded collagen bodies. D. The cells of the proliferation were round, monomorphic, without mitosis or significant atypia 

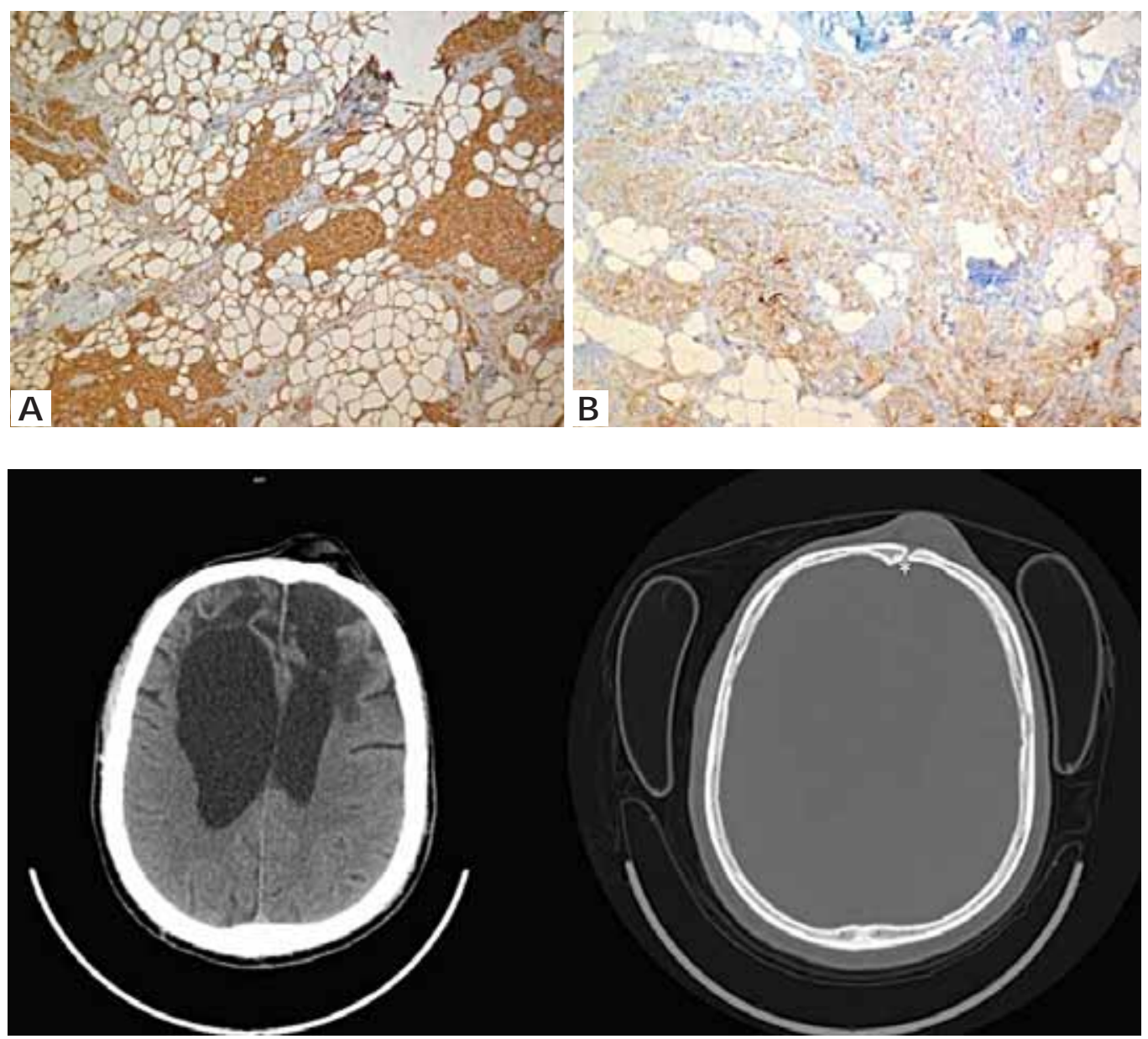

\section{Figure 3:}

Immunohistochemical staining revealed positivity for vimentin (A) and EMA (B)

\section{FIgURE 4:}

Cranioencephalic $\mathrm{CT}$ revealed frontal mass and a bony defect in the frontal bone $\left({ }^{*}\right)$. There were no intracranial masses detected. Frontal encephalomalacia and ventricular asymmetry had been already present in previous exams

\section{DISCUSSION}

Because of its rarity, the literature regarding cutaneous meningiomas is mainly based on case reports.

In 1974, Lopez et $\mathrm{al}^{2}$ developed a classification based on clinical and histopathological criteria. This classification is still widely used, and divides cutaneous meningiomas into 3 types:

Type I are congenital lesions, although they are frequently noticed later in life. They occur on the scalp (occipital area and along the suture lines) and paravertebral regions, and derive from meningothelial cells that become trapped in the dermis and subcutis during development. There may be a connection with the central nervous system (sinus tract), leading to the assumption that type I meningiomas may represent rudimentary meningoceles that have not kept their connection to the central nervous system. ${ }^{3}$

Type II meningiomas are acquired lesions that probably develop from ectopic arachnoid cells that extend to the skin by contiguity, along cranial and spinal nerves. Hence, they are located around sensory organs (eyes, ears, nose and mouth) and along the course of cranial and spinal nerves. There is no associated meningioma of the neuroaxis. ${ }^{1,2}$
Type III are intracranial meningeal tumors that involve the skin by direct extension through bone, traumatic or surgical defects, occurring most commonly on the face, temple and scalp as slow-growing subcutaneous masses. ${ }^{2}$

Due to its rarity and lack of characteristic clinical features (painless subcutaneous nodules, alopecic areas with tufts of hair or hipertrichosis), the differential diagnosis is quite broad, including nevus sebaceous, alopecia areata, adnexal tumors, dermoid cysts, pseudocysts, fibromas, hemangiomas, squamous cell carcinoma, hamartomas, meningocele, meningomyelocele, nasal glioma, neuroectodermic tumours, metastatic lesions. ${ }^{1,2,3,4}$

The clinical features and imaging studies are most of the times inconclusive, making cytology, and most notably histology, crucial for establishing the definitive diagnosis. The most common histopathogical form is the meningothelial type, with lobules, nests and sheets of oval/polygonal meningothelial cells, with a whorled arrangement that shows hyaline structures (collagen bodies) or calcification foci (psammomma bodies), which are very helpful clues to the diagnosis. ${ }^{4}$ 
The immunohistochemical features in our patient's lesion are in agreement with other reported cases: positivity for EMA and vimentin, and negativity for cytokeratins, S100 protein, CD34 and CD31, CD68, desmin and smooth muscle actin. These allow to exclude epithelial, melanocytic, vascular, histiocytic and myogenic differentation, respectively. ${ }^{4}$

The gold standard treatment is complete surgical excision, although some advocate conservative treatment if the lesion is asymptomatic and there are no complications. ${ }^{1,4}$

Type I lesions have better prognosis than type II and III. Some type III lesions can be inoperable, significantly worsening the vital prognosis. Some investigational studies based on molecular-based targeted therapies such as vascular endothelial growth factor inhibitors and platelet-derived growth factor inhibitors are being performed. ${ }^{5}$

\section{REFERENCES}

1. Miedema JR, Zedek D. Cutaneous meningioma. Arch Pathol Lab Med. 2012;136:208-11.

2. Lopez DA, Silvers DN, Helwig EB. Cutaneous meningiomas - a clinicopathologic study. Cancer. 1974;34:728-44.

3. Borggreven PA, de Graaf FH, van der Valk P, Leemans CR. Post-traumatic cutaneous meningioma. J Laryngol 0tol. 2004;118:228-30.

4. Hussein MR, Abdelwahed AR. Primary cutaneous meningioma of the scalp: a case report and review of literature. J Cutan Pathol. 2007;34:26-8.

5. Campbell BA, Jhamb A, Maguire JA, Toyota B, Ma R. Meningiomas in 2009: controversies and challenges. Am J Clin Oncol. 2009;32:73-85.
Although our case does not fit neatly into any specific type of cutaneous meningioma, it is probably best regarded as a variant of a type II lesion. We might speculate that there may be a relation with the previous head trauma, with presumable extension of meningeal tissue through the bone structural defect.

The authors highlight the importance of a detailed clinical history in such cases, which may contribute to evoke the possibility of cutaneous meningioma in certain clinical settings. Histopathological examination plays the major role in establishing the definitive diagnosis, namely through careful assessment of the morphological features combined with a selected panel of immunohistochemical stains. Finally, imaging studies are useful to detect associated features (namely a connection to the neuroaxis or a possible association with an intracranial meningioma), and are of great importance in planning the correct therapeutic approach in these cases.]

H ow to cite this article: Ramos L, Coutinho I, Cardoso JC, Garcia H, Cordeiro MR. Frontal cutaneous meningioma - Case report. An Bras Dermatol. 2015;90 (3 Suppl 1):S130-3. 